[microreview]

Diamond Open Access

\title{
The power set of power sets
}

\author{
Open Mathematics Collaboration*† \\ March 10, 2020
}

\begin{abstract}
We compute $\mathcal{P}(\mathcal{P}(\mathcal{P}(\varnothing)))$, where $\mathcal{P}(\varnothing)$ is the power set of the empty set.
\end{abstract}

keywords: power set, empty set

\section{Definition of the Power Set}

1. If $S$ is a set, then the power set of $S$ is the set that consists of all the subsets of $S[1,2]$.

*All authors with their affiliations appear at the end of this paper.

†Corresponding author: mplobo@uft.edu.br | Join the Open Mathematics Collaboration 


\section{The power set of the power set}

2. $\varnothing=\{\}$

3. Let $\mathcal{P}^{n}(\varnothing)=\mathcal{P}(\mathcal{P}(\ldots \mathcal{P}(\varnothing)))$ have $n$ power sets.

4. $\mathcal{P}^{1}(\varnothing)=\mathcal{P}(\varnothing)=\{\varnothing\}$

5. $\mathcal{P}^{2}(\varnothing)=\mathcal{P}(\mathcal{P}(\varnothing))=\{\varnothing,\{\varnothing\}\}$

6. $\mathcal{P}^{3}(\varnothing)=\mathcal{P}(\mathcal{P}(\mathcal{P}(\varnothing)))=\{\varnothing,\{\varnothing\},\{\{\varnothing\}\},\{\varnothing,\{\varnothing\}\}\}$

\section{Final Remarks}

7. The power set of the power set of the power set of the empty set is

$$
\mathcal{P}(\mathcal{P}(\mathcal{P}(\varnothing)))=\{\varnothing,\{\varnothing\},\{\{\varnothing\}\},\{\varnothing,\{\varnothing\}\}\} .
$$

Note

8.

$$
\begin{aligned}
\mathcal{P}^{4}(\varnothing)= & \mathcal{P}(\mathcal{P}(\mathcal{P}(\mathcal{P}(\varnothing))))= \\
= & \{\varnothing,\{\varnothing\},\{\{\varnothing\}\},\{\varnothing,\{\varnothing\}\}, \\
& \{\varnothing,\{\varnothing\}\},\{\varnothing,\{\{\varnothing\}\}\},\{\varnothing,\{\varnothing,\{\varnothing\}\}\},\{\{\varnothing\},\{\{\varnothing\}\}\}, \\
& \{\{\varnothing\},\{\varnothing,\{\varnothing\}\}\},\{\{\{\varnothing\}\},\{\varnothing,\{\varnothing\}\}\}, \\
& \{\varnothing,\{\varnothing\},\{\{\varnothing\}\}\},\{\varnothing,\{\varnothing\},\{\varnothing,\{\varnothing\}\}\}, \\
& \{\{\varnothing\},\{\{\varnothing\}\},\{\varnothing,\{\varnothing\}\}\}, \\
& \{\varnothing,\{\varnothing\},\{\{\varnothing\}\},\{\varnothing,\{\varnothing\}\}\}
\end{aligned}
$$


9.

$$
\mathcal{P}^{n}=?
$$

10.

$$
\mathcal{P}^{\infty}=\lim _{n \rightarrow \infty} \mathcal{P}^{n}=?
$$

11.

$$
\mathcal{P}^{\omega}=?
$$

12.

$$
\aleph_{\mathcal{P}^{\infty}}=?
$$

13.

$$
\aleph_{\mathcal{P}} \omega=?
$$

14.

$$
\aleph_{\mathcal{P}} \omega>\aleph_{\mathcal{P}} ?
$$

\section{Open Invitation}

Review, add content, and co-author this paper $3,4.4$.

Join the Open Mathematics Collaboration.

Send your contribution to mplobo@uft.edu.br.

\section{Ethical conduct of research}

This original work was pre-registered under the OSF Preprints [5], please cite it accordingly [6]. This will ensure that researches are conducted with integrity and intellectual honesty at all times and by all means.

\section{References}

[1] Simovici, Dan, and Richard L. Tenney. Theory of formal languages with applications. World Scientific Publishing Company, 1999. 
[2] Velleman, Daniel J. How to prove it: A structured approach. Cambridge University Press, 2019.

[3] Lobo, Matheus P. "Microarticles." OSF Preprints, 28 Oct. 2019. https://doi.org/10.31219/osf.io/ejrct

[4] Lobo, Matheus P. "Simple Guidelines for Authors: Open Journal of Mathematics and Physics." OSF Preprints, 15 Nov. 2019. https://doi.org/10.31219/osf.io/fk836

[5] COS. Open Science Framework. https://osf.io

[6] Lobo, Matheus P. "The Power Set of Power Sets." OSF Preprints, 8 Mar. 2020. https://doi.org/10.31219/osf.io/v5zyb

\section{The Open Mathematics Collaboration}

Matheus Pereira Lobo (lead author, mplobo@uft.edu.br) $)^{1,2}$

${ }^{1}$ Federal University of Tocantins (Brazil); ${ }^{2}$ Universidade Aberta (UAb, Portugal) 